\title{
Peso de Abate de Machos Não-Castrados para Produção do Bovino Jovem. 2. Peso, Idade e Características da Carcaça
}

\author{
Geraldo Maria da Cruz ${ }^{1}$, Rymer Ramiz Tullio², Sérgio Novita Esteves ${ }^{3}$, \\ Maurício Mello de Alencar ${ }^{4}$, César Antônio Cordeiro ${ }^{5}$
}

\begin{abstract}
RESUMO - Com o objetivo de obter o peso e idade de abate e as características de carcaça de bovinos machos não-castrados, o confinamento foi desenvolvido na Embrapa Pecuária do Sudeste. Um total de 215 animais, da Embrapa e de produtores particulares, sendo 53 Blonde d'Aquitaine x Nelore (BN), 36 Canchim x Nelore (CN), 36 Limousin x Nelore (LN), 36 Piemontês x Nelore (PN), 18 Canchim (CA) e 36 Nelore (NE), foram utilizados. Foram avaliados os pesos de abate (TRAT) de 400 (I), 440 (II) e 480 kg (III), exceto para os bovinos NE, que foram de 380, 410 e $440 \mathrm{~kg}$. Os animais receberam uma dieta, ad libitum, à base de $50 \%$ de silagem de milho e $50 \%$ de concentrado, na base seca. O peso vivo dos animais foi obtido, após jejum de água e alimentos de 16 horas. Os animais foram abatidos em frigorífico comercial. Os dados foram submetidos à análise de variância e as médias, comparadas pelo teste SNK. Os animais cruzados e Canchim foram abatidos com 231, 252 e $273 \mathrm{~kg}$ de carcaça quente aos 15,4; 16,1 e 16,8 meses de idade para os TRAT I, II e III, respectivamente, enquanto as carcaças dos animais NE pesaram 211,5; 219 e $228 \mathrm{~kg}$ aos 16,4; 17,2 e 17,5 meses, respectivamente. As médias estimadas de período experimental, rendimento de carcaça, área de olho de lombo e espessura de gordura externa foram: 70,9; 95,1 e 114,7 dias; 57,2; 57,7 e 58,3\%; 31,4; 30,9 e 30,2 cm²/100 kg de carcaça; 2,5; 3,1 e 3,6 mm, para os TRAT I, II e III, respectivamente. A percentagem de carne comestível do traseiro especial não foi alterada pelos pesos de abate, ocorrendo redução na percentagem de ossos e aumento na percentagem de aparas de gordura, à medida que aumentou o peso de abate. Ocorreram interações entre TRAT e grupos genéticos para pesos estimados de abate, percentagens de cortes traseiro total, traseiro especial e dianteiro da carcaça resfriada e área de olho de lombo.
\end{abstract}

Palavras-chave: área de olho de lombo, confinamento, cruzados, desossa, espessura de gordura externa, grupo genético

\section{Slaughter Weights for the Production of Young Bull Cattle. 2. Weight, Age and Carcass Characteristics}

\begin{abstract}
To obtain slaughter weight and age and the carcass characteristics of young bulls, a feedlot study was conducted at Embrapa Pecuária Sudeste. A total of 215 animals from Embrapa and private farms, $3 / 8$ and 1 1 2 Blonde d'A Auitaine x Nellore; $1 / 2$ Canchim x Nellore; $1 \frac{1}{2}$ Limousin x Nellore; 1/2 Piedmontese x Nellore; Canchim (CA) and Nellore (NE), were used in 1994, 1995 and 1997. The slaughter weights (TRAT) of 400 (I); 440 (II) and $480 \mathrm{~kg}$ (III), except for NE cattle (380, 410 and $440 \mathrm{~kg}$ ), were tested. All animals received, ad libitum, a diet based on 50\% whole plant corn silage and 50\% concentrate, on a dry matter basis. The live weight of the animals were obtained after fasting of 16 hours. The animals were slaughtered at a commercial packing plant. The data were submitted to the analysis of variance and the means were compared by SNK tests. The straightbred Canchim and all crossbred animals were slaughtered at 231, 252 and $273 \mathrm{~kg}$ of hot carcass weight with an average age of 15.4; 16.1 and 16.8 months, for TRAT I, II and III, respectively; while straightbred Nellore were slaughtered at 211.5; 219 and $228 \mathrm{~kg}$ hot carcass weight with an average age of 16.4; 17.2 and 17.5 months, for TRAT I, II and III, respectively. The estimated means of days on feedlot, yield of carcass, rib eye area and backfat thickness were: 70.9; 95.1 and 114.7 days; 57.2; 57.7 and 58.3\%; 31.4; 30.9 and $30.2 \mathrm{~cm}^{2} / 100 \mathrm{~kg}$ carcass weight; 2.5 ; 3.1 and 3.6 mm, for TRAT I, II and III, respectively. The percentage of saleable meat from the hindquarter gunbit cut was similar for all three slaughter weights; while a reduction of the percentage of bones was compensated for by an increase in the percentage of trimmed excess fat with an increase of slaughter weight. There were significant interactions between genetic group and TRAT for estimated slaughter weights, percentage of hindquarter, hindquarter gunbit and forequarter cuts and the rib eye area.
\end{abstract}

Key Words: backfat thickness, crossbred, cutability, feedlot, genetic group, rib eye area

\footnotetext{
${ }_{1}^{1}$ Pesquisador da Embrapa Pecuária Sudeste, C.P. 339, 13560-970, São Carlos, SP. E.mail: geraldo@cppse.embrapa.br

${ }^{2}$ Pesquisador da Embrapa Pecuária Sudeste, pós-graduando em Zootecnia FCAVIUNESP, Jaboticabal, SP.

3 Pesquisador da Embrapa Pecuária Sudeste. E.mail: sergio@cppse.embrapa.br

${ }^{4}$ Pesquisador da Embrapa Pecuária Sudeste. E.mail: mauricio@cppse.embrapa.br. Bolsista CNPq

${ }^{5}$ Técnico Especializado II da Embrapa Pecuária Sudeste. E.mail: cesar@cppse.embrapa.br
} 


\section{Introdução}

A técnica de confinamento de bovinos é utilizada com sucesso em todo mundo, no intuito de intensificar a produção de carne bovina, ou como alternativa a produção de carne, em períodos de escassez de forragem. Na década de 70, nos países desenvolvidos, já era recomendado o abate de machos com menos de 18 meses de idade (Preston \& Willis, 1974), mas no Brasil as dificuldades para abater animais jovens ainda persistem.

A carne bovina produzida no Brasil é considerada de qualidade inferior, devido ao fato de o País produzir gado Bos taurus indicus para abate. A carne de zebuínos puros foi considerada dura (Francis et al., 1981; Abullarach et al., 1998) e apresentou redução de palatabilidade com o aumento do "grau de sangue" de zebu nos cruzamentos com bovinos de raças européias (Crouse et al., 1989). Segundo Felício (1997), o segmento da produção de bovinos pode ter um papel importante na melhoria da qualidade organoléptica da carne, utilizando o melhoramento genético para características produtivas e reprodutivas, e adotando sistemas de manejo e alimentação que possibilitem o abate de tourinhos entre 18 e 24 meses de idade apresentando peso de carcaça compatível com as necessidades mínimas de acabamento.

O sistema americano de classificação e tipificação de carcaças de bovinos possui um componente importante - a gordura intramuscular (marmorização). No sistema brasileiro de tipificação de carcaças (Sainz \& Araujo, 2001), a gordura externa é o principal parâmetro para detectar o ponto de abate. Este fator, que também é importante no sistema americano, é indispensável para conferir ao produto condições mínimas de manuseio e palatabilidade, sendo também responsável pela redução da velocidade de resfriamento das carcaças.

Conforme Perotto et al. (2000), o aumento do peso e a melhoria da qualidade das carcaças estão entre os benefícios que os cruzamentos entre raças Bos taurus taurus e Bos taurus indicus proporcionam, de forma imediata, à pecuária bovina de corte. Euclides Filho et al. (1997a) afirmam que os cruzamentos têm mostrado ser boa alternativa para maior inserção da pecuária de corte brasileira em um mercado de carne cuja tendência é tornar-se cada vez mais competitivo, no qual a qualidade da carne desempenha um papel de fundamental importância. Dessa forma, a utilização de cruzamentos vem cres- cendo na expansão e modernização dos sistemas de produção.

O peso de abate pode ser fator importante na determinação da qualidade da carcaça. Barber et al. (1981) encontraram aumento do rendimento de carcaça e redução no rendimento de cortes nobres, com o incremento do peso de abate, segundo o sistema americano de desossa. No Brasil, Galvão et al. (1991) encontraram melhoria no rendimento de carcaça quente e na deposição de gordura, com o aumento do peso de abate. Este estudo foi conduzido com o objetivo de comparar os pesos de abate e as características de carcaça de machos jovens não-castrados, abatidos aos 400, 440 ou $480 \mathrm{~kg}$ de peso vivo para animais cruzados e puros Canchim e 380, 410 e 440 para animais Nelore.

\section{Material e Métodos}

O trabalho foi desenvolvido na Embrapa Pecuária Sudeste, em 1994, 1995 e 1997 com 53 animais cruzados $1 / 2(3 / 8)$ Blonde d'Aquitaine $x 1 / 2(5 / / 8)$ Nelore (BN); $361 \frac{1}{2}$ Canchim x $1 / 2$ Nelore (CN), 36 1/2 Limousin $x$ 1/2 Nelore (LN) e 36 1/2 Piemontês x 1/2 Nelore (PN) e 18 puros Canchim (CA) e 36 Nelore (NE), sendo que os grupos genéticos CN, LN, PN e $\mathrm{NE}$ participaram em dois anos, $\mathrm{BN}$ em três anos e CA em um ano dos estudos de qualidade de carcaça. Os 17 animais BN do ano de 1994 eram 3/8 Blonde d'Aquitaine x 5/ ${ }_{8}$ Nelore, enquanto, em 1995 e 1997, foram utilizados animais $1 / 2$ Blonde d'Aquitaine $x 1 / 2$ Nelore. Os animais CA e CN pertenciam ao rebanho da Embrapa Pecuária Sudeste, enquanto os animais dos outros grupos genéticos pertenciam a rebanhos de produtores particulares, de propriedades localizadas nos municípios de Marabá, PA e Avaré, Bariri e Santa Fé do Sul, SP. Lotes de seis animais de cada GG foram aleatoriamente alocados nos tratamentos (TRAT), que são os pesos de abate de 400 (I), 440 (II) e $480 \mathrm{~kg}$ (III), exceto para os bovinos NE, que foram de 380 (I), 410 (II) e $440 \mathrm{~kg}$ (III). Um animal BN foi sacrificado após um acidente de manejo no final do período de adaptação. Os animais receberam, ad libitum, uma dieta com 13\% de proteína bruta (PB) e $70 \%$ de nutrientes digestíveis totais (NDT), à base de $50 \%$ de silagem de milho, 29,2\% de milho em grão moído, 9,1\% de farelo de soja, 9,9\% de farelo de trigo, $0,8 \%$ de calcário calcítico e $1 \%$ de mistura mineral, na base seca. O peso de abate dos animais no confinamento foi obtido a cada 28 dias, precedido

R. Bras. Zootec., v.33, n.3, p.646-657, 2004 
de um período pré-experimental de 31, 27 e 36 dias, nos anos de 94, 95 e 97, respectivamente, após jejum de água e alimentos de 16 horas, inclusive antes dos abates. Os pesos de carcaça quente, da gordura peri-renal, pélvica e inguinal (gordura interna), dos cortes da carcaça resfriada (traseiro especial, dianteiro com cinco costelas e ponta de agulha) foram obtidos no frigorífico.

O traseiro esquerdo especial (TEE), de cada animal foi dividido entre a $12^{\mathrm{a}}$ e a $13^{\mathrm{a}}$ costela para a medição da área de olho de lombo (AOL) e da espessura de gordura externa (EGAOL). Em seguida, realizou-se a desossa tradicional do referido traseiro com a obtenção dos cortes: filé mignon, contrafilé, alcatra completa, capa e aba do contrafilé, coxão mole, coxão duro, patinho, lagarto e músculo. Após a "limpeza" dos cortes acima, obtiveram-se os pesos dos respectivos cortes, dos retalhos (aparas) de carne e gordura e dos ossos. A porção comestível do TEE foi obtida pela soma dos pesos dos nove cortes cárneos mais as aparas de carne, expressando o resultado em percentagem do peso do TEE.

Os dados de peso de abate, idade de abate e de características de carcaça foram submetidos à análise de variância pelo procedimento GLM do SAS (1999), considerando os efeitos de Ano, GG, TRAT e a interação GG x TRAT. Como a interação GG x TRAT foi significativa para grande número das variáveis testadas, também foi aplicado o modelo com as variáveis ANO e TRAT para cada GG, separadamente. As diferentes médias foram comparadas pelo teste SNK.

\section{Resultados e Discussão}

As médias estimadas de peso de abate, peso de carcaça quente, percentagem de cortes da carcaça resfriada, peso do traseiro esquerdo especial (TEE) e área de olho de lombo (AOL), por tratamento, para os grupos genéticos BN, CN, CA, LN, PN e NE estão apresentadas separadamente nas Tabelas 1 a 6 , respectivamente. As interações significativas $(\mathrm{P}<0,05)$ GG x TRAT para as variáveis peso de abate, peso de carcaça quente e peso do traseiro especial, provavelmente, ocorreram em função da presença de animais $\mathrm{NE}$, que apresentaram taxa de ganho de peso abaixo do esperado ao final do confinamento, provocando abate antecipado de vários animais deste grupo genético, em relação às metas de peso de abate de 410 e $440 \mathrm{~kg}$. As interações significativas $(\mathrm{P}<0,05) \mathrm{GG}$ $\mathrm{X}$ TRAT para as porcentagens de traseiro total, traseiro especial e dianteiro e área de olho de lombo, continuaram presentes mesmo com a retirada dos dados dos animais NE do arquivo. No caso das percentagens dos diversos cortes secundários da carcaça, ocorreram tendências ou até reduções significativas $(\mathrm{P}<0,05)$ na porcentagem de traseiro e aumento de dianteiro, com os incrementos nos pesos de abate nos grupos genéticos BN, CA, CN e LN, ao contrário de PN e NE, que não apresentaram tal tendência.

De maneira geral, foi possível abater animais aos 231, 252 e $273 \mathrm{~kg}$ de peso de carcaça quente aos 15,5; 16,3 e 16,9 $\pm 0,15$ meses de idade, para os TRAT I, II e III, respectivamente, considerando a média dos resultados dos animais CA, BN, CN, LN e PN. Já os animais do grupo NE foram abatidos com 211,5; 219 e $228 \mathrm{~kg}$ aos 16,4; 17,2 e 17,5 \pm 0,39 meses de idade. Broadbent (1976) abateu animais com 240,5; 266,2 e $295,7 \mathrm{~kg}$ de carcaça quente aos 14,4; 16,8 e 18,2 meses de idade. Costa et al. (2002) abateram novilhos Angus com 181; 203; 211 e 239 kg de carcaça resfriada aos 12, 13, 14 e 15 meses de idade. Huffman et al. (1990) abateram animais cruzados Angus x Brahman com $300 \mathrm{~kg}$ de peso de carcaça aos 17 meses de idade, enquanto Euclides Filho et al. (1997b) abateram animais, não-castrados, Nelore e cruzados 3/4 Nelore x 1/4 Charolês; 3/4 Nelore x $1 / 4$ Fleckvieh e 3/4 Nelore $x$ 1/4 Chianina aos $450 \mathrm{~kg}$ de peso vivo e $265 \mathrm{~kg}$ de carcaça, confinados a partir de 11 meses de idade, e obtiveram idade de abate de 18 meses. Euclides Filho et al. (1997c) abateram animais da raça Nelore com suplementação a pasto na primeira seca e confinados por 100 dias na segunda seca, com peso de $440 \mathrm{~kg}$ e 21,5 meses de idade. A maioria dos animais do grupo NE do presente estudo não atingiu os pesos previstos para abate de $410 \mathrm{~kg}$ (II) e $440 \mathrm{~kg}$ (III), em razão do baixo ganho de peso $(1,11 \pm 0,06 \mathrm{~kg} / \mathrm{dia})$ e do baixo peso vivo na entrada do confinamento (213,9 $\pm 3,8 \mathrm{~kg}$ ). Contudo, ao final do período experimental, estes animais apresentavam-se com terminação de gordura adequada, pela avaliação visual. O mesmo fato ocorreu com um animal BN (TRAT III), dois CN (TRAT II e III), um CA (TRAT III) e um PN (TRAT III).

Todos os 215 animais abatidos possuíam dentição de leite dentro do padrão de idade previsto na classificação de "Novilho Precoce", para abate de machos não-castrados (Mattos, 1995; Pires, 1995; Sainz \& Araujo, 2001). Outro fator importante na classificação do bovino como "Novilho Precoce" é o peso de carcaça quente. No Estado de Minas Gerais (Pires,

\section{R. Bras. Zootec., v.33, n.3, p.646-657, 2004}


Tabela 1 - Médias estimadas do peso de abate e características de carcaça de animais não-castrados Blonde d'Aquitaine x Nelore, abatidos aos 400 (I), 440 (II) e 480 (III) $\mathrm{kg}^{1}$

Table 1 - Estimated means of slaughter weight and carcass characteristics of bulls Blonde $d^{\prime}$ Aquitaine $x$ Nellore slaughtered at 400 (I), 440 (II) and 480 (III) $\mathrm{kg}^{1}$

\begin{tabular}{|c|c|c|c|c|}
\hline & \multicolumn{3}{|c|}{$\begin{array}{l}\text { Peso de abate, } \mathrm{kg} \\
\text { Slaughter weight, } \mathrm{kg}\end{array}$} & \multirow[b]{2}{*}{$\begin{array}{l}\text { Erro-padrão } \\
\text { Standard error }\end{array}$} \\
\hline & 400 & 440 & 480 & \\
\hline $\begin{array}{l}\text { Peso de abate, } \mathrm{kg} \\
\text { Slaughter weight, } \mathrm{kg}\end{array}$ & $399,8^{\mathrm{C}}$ & $438,8^{b}$ & $470,7^{a}$ & 4,0 \\
\hline $\begin{array}{l}\text { Peso de carcaça quente, } \mathrm{kg} \\
\text { Hot carcass weight, } \mathrm{kg}\end{array}$ & $230,9^{c}$ & $259,2^{b}$ & $274,5^{\mathrm{a}}$ & 2,7 \\
\hline $\begin{array}{l}\text { Dias em confinamento } \\
\text { Days on feedlot }\end{array}$ & $59,1^{\mathrm{C}}$ & $83,3^{\mathrm{b}}$ & $101,9^{\mathrm{a}}$ & 4,2 \\
\hline $\begin{array}{l}\text { Percentagem de traseiro especial } \\
\text { Yield of hindquarter gun cut, } \%\end{array}$ & $48,1^{\mathrm{a}}$ & $47,7^{\mathrm{ab}}$ & $47,2^{\mathrm{b}}$ & 0,23 \\
\hline $\begin{array}{l}\text { Percentagem de dianteiro } \\
\text { Yield of forequarter cut, } \%\end{array}$ & $39,3^{\mathrm{a}}$ & $39,5^{\mathrm{a}}$ & $39,8^{\mathrm{a}}$ & 0,27 \\
\hline $\begin{array}{l}\text { Percentagem de ponta de agulha } \\
\text { Yield of flank with ribs cut, \% }\end{array}$ & $12,6^{\mathrm{a}}$ & $12,7^{\mathrm{a}}$ & $12,9^{\mathrm{a}}$ & 0,15 \\
\hline $\begin{array}{l}\text { Percentagem de traseiro total } \\
\text { Yield of hindquarter cut, \% }\end{array}$ & $60,7^{\mathrm{a}}$ & $60,5^{\mathrm{a}}$ & $60,2^{\mathrm{a}}$ & 0,26 \\
\hline $\begin{array}{l}\text { Traseiro esquerdo especial, kg } \\
\text { Left hindquarter gunbit cut, } \mathrm{kg}\end{array}$ & $54,0^{\mathrm{C}}$ & $60,4^{\mathrm{b}}$ & $63,5^{a}$ & 0,57 \\
\hline $\begin{array}{l}\text { Área de olho de lombo, } \mathrm{cm}^{2} \\
\text { Rib eye area, } \mathrm{cm}^{2}\end{array}$ & $74,7^{\mathrm{b}}$ & $81,3^{\mathrm{a}}$ & $82,2^{\mathrm{a}}$ & 1,88 \\
\hline
\end{tabular}

1995), é aceito bovino macho não-castrado, com dentição de leite, com peso de carcaça acima de $200 \mathrm{~kg}$ e em outros Estados, acima de $225 \mathrm{~kg}$. Com base neste parâmetro, os animais Nelore acima de $380 \mathrm{~kg}$ de peso vivo seriam classificados como "Novilho Precoce" em Minas Gerais e acima de 410 kg nos outros Estados. No presente trabalho, pode ser observado que animais NE com peso vivo de $215 \mathrm{~kg}$ aos 12 meses de idade necessitam de um período total de confinamento de 150 dias em Minas Gerais e de 180 dias em outros Estados. Já os animais dos outros cinco grupos genéticos não possuem limitações de ganho de peso e peso vivo no início do confinamento, para atingirem peso mínimo de abate. Mais recentemente, Sainz \& Araujo (2001) relatam que se encontra no início a política de diferenciação de preços, de acordo com o tipo de carcaça, nas empresas que utilizam padrões de tipificação próprios e, geralmente, exigem um peso mínimo de carcaça de 16 arrobas. No presente estudo, animais cruzados, confinados aos 12 meses de idade com dieta de silagem de milho e concentrado atingiram a meta estabelecida pela indústria frigorífica aos 16,3 meses de idade e, aproximadamente, 120 dias de período total de confinamento.

A divisão da carcaça resfriada nos cortes traseiro especial, dianteiro com cinco costelas, ponta de agulha e traseiro total (traseiro especial + ponta de agulha) resultou na redução de 0,9 unidades de percentagem no traseiro especial e aumentos de 0,5 e 0,4 unidades de percentagem no dianteiro e ponta de agulha, respectivamente, com o aumento de peso de abate de 400 para $480 \mathrm{~kg}$. Contudo, foi observada interação $(P<0,05)$ GG x TRAT. Esta interação se deve provavelmente à maior precocidade sexual, com desenvolvimento de características sexuais secundárias, em alguns grupos genéticos, e também a diferenças entre idades de abate dos diferentes grupos genéticos, que, apesar de apresentarem adequadas para produção do "Novilho Precoce", podem ter sido suficiente para causar a interação. Os animais PN e NE não apresentaram mudanças nas percentagens dos diferentes cortes com o aumento de peso de abate. Galvão et al. (1991) e Jorge et al. (1997b) não encontraram 
Tabela 2 - Médias estimadas do peso de abate e características de carcaça de animais não-castrados Canchim, abatidos aos 400 (I), 440 (II) e 480 (III) kg ${ }^{1}$

Table 2 - Estimated means of slaughter weight and carcass characteristics of Canchim bulls slaughtered at 400 (I), 440 (II) and 480 (III) kg ${ }^{1}$

\begin{tabular}{|c|c|c|c|c|}
\hline & \multicolumn{3}{|c|}{$\begin{array}{l}\text { Peso de abate, } \mathrm{kg} \\
\text { Slaughter weight, } \mathrm{kg}\end{array}$} & \multirow[b]{2}{*}{$\begin{array}{l}\text { Erro-padrão } \\
\text { Standard error }\end{array}$} \\
\hline & 400 & 440 & 480 & \\
\hline $\begin{array}{l}\text { Peso de abate, } \mathrm{kg} \\
\text { Slaughter weight, } \mathrm{kg}\end{array}$ & $413,5^{c}$ & $437,7^{b}$ & $465,5^{a}$ & 7,9 \\
\hline $\begin{array}{l}\text { Peso de carcaça quente, } \mathrm{kg} \\
\text { Hot carcass weight, } \mathrm{kg}\end{array}$ & $229,8^{c}$ & $247,0^{\mathrm{b}}$ & $264,1^{\mathrm{a}}$ & 5,0 \\
\hline $\begin{array}{l}\text { Dias em confinamento } \\
\text { Days on feedlot }\end{array}$ & $55,4^{b}$ & $87,4^{\mathrm{a}}$ & $109,5^{\mathrm{a}}$ & 5,3 \\
\hline $\begin{array}{l}\text { Percentagem de traseiro especial } \\
\text { Yield of hindquarter gun cut, \% }\end{array}$ & $48,1^{\mathrm{a}}$ & $47,9^{a}$ & $46,9^{a}$ & 0,46 \\
\hline $\begin{array}{l}\text { Percentagem de dianteiro } \\
\text { Yield of forequarter cut, } \%\end{array}$ & $38,8^{a}$ & $38,7^{\mathrm{a}}$ & $39,2^{\mathrm{a}}$ & 0,38 \\
\hline $\begin{array}{l}\text { Percentagem de ponta de agulha } \\
\text { Yield of flank with ribs cut, \% }\end{array}$ & $13,0^{\mathrm{a}}$ & $13,4^{\mathrm{a}}$ & $14,0^{\mathrm{a}}$ & 0,31 \\
\hline $\begin{array}{l}\text { Percentagem de traseiro total } \\
\text { Yield of hindquarter cut, \% }\end{array}$ & $61,1^{\mathrm{a}}$ & $61,3^{a}$ & $60,8^{a}$ & 0,38 \\
\hline $\begin{array}{l}\text { Traseiro esquerdo especial, } \mathrm{kg} \\
\text { Left hindquarter gunbit cut, } \mathrm{kg}\end{array}$ & $54,2^{b}$ & $58,4^{\mathrm{a}}$ & $61,3^{a}$ & 1,32 \\
\hline $\begin{array}{l}\text { Área de olho de lombo, } \mathrm{cm}^{2} \\
\text { Rib eye area, } \mathrm{cm}^{2}\end{array}$ & $78,1^{a}$ & $77,6^{\mathrm{a}}$ & $80,0^{\mathrm{a}}$ & 3,41 \\
\hline
\end{tabular}

interações entre grupo genético e pesos de abate para as percentagens dos cortes da carcaça. As percentagens de traseiro especial, em relação ao peso da carcaça resfriada, sofreram redução $(\mathrm{P}<0,05)$ nos grupos genéticos $\mathrm{BN}, \mathrm{CN}$ e LN, com o aumento do peso de abate, sendo que nos animais CA ocorreu a mesma tendência, porém não significativa $(\mathrm{P}>0,05)$, provavelmente em razão do menor número de animais abatidos deste GG. Costa et al. (2002) encontraram redução na percentagem do traseiro especial em relação ao peso total da carcaça resfriada, com o aumento de peso de abate de 340 para 370, 400 ou $430 \mathrm{~kg}$, estando de acordo com os resultados de Galvão et al. (1991) e Broadbent (1976), que também observaram redução de 46,7 para 45,4 ou $44,9 \%$ com o aumento do peso de abate de 418 para 459 ou $496 \mathrm{~kg}$. Entretanto, Jorge et al. (1997b) não encontraram diferenças nas percentagens de traseiro especial com o aumento de peso de abate de 500 para $550 \mathrm{~kg}$ para os animais cruzados e de 450 para $500 \mathrm{~kg}$ para nelore.

As médias estimadas de percentagem do traseiro total apresentaram redução nos grupos genéticos BN, CA, CN e LN, sendo significativa $(\mathrm{P}<0,05)$ apenas no grupo LN. A média estimada de traseiro total, para todos os GG, foi de $60,6 \pm 0,13 \%$, dentro dos padrões adequados para produção de uma carcaça com alto valor comercial, já que os principais cortes comerciais (preços mais elevados) estão contidos no traseiro. Galvão et al. (1991) e Jorge et al. (1997b) não encontraram diferenças nas percentagens de traseiro total com o aumento do peso de abate.

A análise do peso do TEE, à semelhança do peso de carcaça quente, mostrou interação significativa $(\mathrm{P}<0,05)$ entre GG e TRAT. O peso do TEE aumentou $(\mathrm{P}<0,05)$ com o incremento no peso de abate, em todos os GG de animais cruzados e CA. As diferenças de peso de abate obtidas para o grupo NE foram muito pequenas; dessa maneira, os pesos do TEE foram semelhantes. Os valores obtidos para os pesos do TEE dos animais cruzados e CA dos TRAT II e III (Tabelas 1 a 5) estão próximos da exigência do mercado para traseiro especial (serrote), ou seja, peso de 60 a $65 \mathrm{~kg}$.

As médias estimadas das variáveis que não apresentaram interação significativa $(\mathrm{P}<0,05) \mathrm{GG}$ x TRAT, quais sejam: idade de abate, período de confinamento, percentagem de gordura interna (peri-renal, pélvica, 
Tabela 3 - Médias estimadas do peso de abate e características de carcaça de animais não-castrados Canchim x Nelore, abatidos aos 400 (I), 440 (II) e 480 (III) $\mathrm{kg}^{1}$

Table 3 - Estimated means of slaughter weight and carcass characteristics of bulls Canchim $x$ Nellore slaughtered at 400 (I), 440 (II) and 480 (III) $\mathrm{kg}^{1}$

\begin{tabular}{|c|c|c|c|c|}
\hline & \multicolumn{3}{|c|}{$\begin{array}{l}\text { Peso de abate, } \mathrm{kg} \\
\text { Slaughter weight, } \mathrm{kg}\end{array}$} & \multirow[b]{2}{*}{$\begin{array}{l}\text { Erro-padrão } \\
\text { Standard error }\end{array}$} \\
\hline & 400 & 440 & 480 & \\
\hline Peso de abate, kg & $404,1^{\mathrm{c}}$ & $427,8^{b}$ & $458,6^{\mathrm{a}}$ & 5,8 \\
\hline Slaughter weight, $\mathrm{kg}$ & & & & \\
\hline $\begin{array}{l}\text { Peso de carcaça quente, } \mathrm{kg} \\
\text { Hot carcass weight, } \mathrm{kg}\end{array}$ & $227,0^{\mathrm{C}}$ & $241,6^{b}$ & $267,0^{\mathrm{a}}$ & 3,8 \\
\hline $\begin{array}{l}\text { Dias em confinamento } \\
\text { Days on feedlot }\end{array}$ & $58,4^{\mathrm{b}}$ & $88,2^{\mathrm{a}}$ & $102,1^{\mathrm{a}}$ & 5,9 \\
\hline $\begin{array}{l}\text { Percentagem de traseiro especial } \\
\text { Yield of hindquarter gun cut, \% }\end{array}$ & $48,0^{\mathrm{a}}$ & $47,4^{\mathrm{ab}}$ & $46,8^{\mathrm{b}}$ & 0,30 \\
\hline $\begin{array}{l}\text { Percentagem de dianteiro } \\
\text { Yield of forequarter cut, } \%\end{array}$ & $38,6^{\mathrm{a}}$ & $39,3^{\mathrm{a}}$ & $39,5^{a}$ & 0,29 \\
\hline $\begin{array}{l}\text { Percentagem de ponta de agulha } \\
\text { Yield of flank with ribs cut, \% }\end{array}$ & $13,3^{\mathrm{a}}$ & $13,3^{\mathrm{a}}$ & $13,7^{\mathrm{a}}$ & 0,21 \\
\hline $\begin{array}{l}\text { Percentagem de traseiro total } \\
\text { Yield of hindquarter cut, \% }\end{array}$ & $61,4^{\mathrm{a}}$ & $60,7^{\mathrm{a}}$ & $60,5^{\mathrm{a}}$ & 0,29 \\
\hline $\begin{array}{l}\text { Traseiro esquerdo especial, kg } \\
\text { Left hindquarter gunbit cut, } \mathrm{kg}\end{array}$ & $53,0^{\mathrm{C}}$ & $56,5^{b}$ & $61,1^{\mathrm{a}}$ & 0,92 \\
\hline $\begin{array}{l}\text { Área de olho de lombo, } \mathrm{cm}^{2} \\
\text { Rib eye area, } \mathrm{cm}^{2}\end{array}$ & $68,0^{\mathrm{b}}$ & $71,8^{b}$ & $76,6^{\mathrm{a}}$ & 1,56 \\
\hline
\end{tabular}

inguinal), rendimento de carcaça quente e resfriada, EGAOL, AOL (expressa em $\mathrm{cm}^{2} / 100 \mathrm{~kg}$ de carcaça), percentagens de carne comestível, de ossos e de aparas de gorduras da desossa do traseiro especial, estão apresentada na Tabela 7.

As médias estimadas de rendimento de carcaça quente foram de 57,$2 ; 57,7$ e $58,3 \pm 0,19 \%$ e de carcaça resfriada, de 56,8; 57,1 e 57,5 \pm 0,19\%, para os tratamentos I, II e III, respectivamente. Com os incrementos dos pesos de abate, ocorreram aumentos significativos $(\mathrm{P}<0,05)$ nos rendimentos de carcaça quente entre os tratamentos II e III e resfriada entre os tratamentos I e III. Resultados semelhantes foram obtidos por Breidenstein et al. (1965), citados por Preston \& Willis (1974), com o aumento do peso de abate de 307 para 386, 466 ou 545 kg, e por Bailey et al. (1985), trabalhando com bovinos machos nãocastrados da raça Holandesa, variando os pesos de abate de 340 a $600 \mathrm{~kg}$. No entanto, Patterson et al. (1994) encontraram aumento linear no rendimento de carcaça quente entre 550 e $700 \mathrm{~kg}$ de peso vivo com machos cruzados, não-castrados, de raças continentais de gado de corte. Galvão et al. (1991) também encontraram aumentos nos rendimentos de carcaça quente, com o aumento de peso de abate de 90 para 100 ou $110 \%$ do peso adulto da raça Nelore (405, 450 e $500 \mathrm{~kg}$ ) e dos cruzados Nelore x Marchigiana e Nelore x Limousin (450, 500 e 550 kg). Huffman et al. (1990) encontraram aumentos de rendimento de carcaça quente entre os pesos de 440 e $475 \mathrm{~kg}$; contudo, aumentando o peso para $507 \mathrm{~kg}$, não houve melhora no rendimento de carcaça de animais da raça Angus e cruzados Angus x Brahman. Entretanto, outros autores não encontraram aumento no rendimento de carcaça quente de bovinos machos nãocastrados da raça Holandesa, com o aumento do peso de abate de 400 para 500 kg (Levy et al., 1975) e de bubalinos e bovinos das raças Nelore e cruzados Nelore x Holandês e bimestiços Fleckvieh x Angus x Nelore com aumento do peso de abate de 500 para $550 \mathrm{~kg}$ para os cruzados e de 450 para $500 \mathrm{~kg}$ para Nelore e búfalos (Jorge et al., 1997b).

As médias estimadas das porcentagens de gordura interna (gordura pélvica, peri-renal e inguinal), expressa em relação ao peso de carcaça quente, aumentaram $(\mathrm{P}<0,05)$ de 3,2 para 3,6 e 3,7 $\pm 0,09 \%$

R. Bras. Zootec., v.33, n.3, p.646-657, 2004 
Tabela 4 - Médias estimadas do peso de abate e características de carcaça de animais não-castrados Limousin x Nelore, abatidos aos 400 (I), 440 (II) e 480 (III) $\mathrm{kg}^{1}$

Table 4 - Estimated means of slaughter weight and carcass characteristics of bulls Limousin $x$ Nellore slaughtered at 400 (I), 440 (II) and 480 (III) $\mathrm{kg}^{1}$

\begin{tabular}{|c|c|c|c|c|}
\hline & \multicolumn{3}{|c|}{$\begin{array}{l}\text { Peso de abate, } \mathrm{kg} \\
\text { Slaughter weight, } \mathrm{kg}\end{array}$} & \multirow[b]{2}{*}{$\begin{array}{l}\text { Erro-padrão } \\
\text { Standard error }\end{array}$} \\
\hline & 400 & 440 & 480 & \\
\hline $\begin{array}{l}\text { Peso de abate, } \mathrm{kg} \\
\text { Slaughter weight, } \mathrm{kg}\end{array}$ & $405,3^{\mathrm{c}}$ & $440,3^{b}$ & $481,2^{\mathrm{a}}$ & 4,6 \\
\hline $\begin{array}{l}\text { Peso de carcaça quente, } \mathrm{kg} \\
\text { Hot carcass weight, } \mathrm{kg}\end{array}$ & $233,2^{\mathrm{C}}$ & $257,8^{\mathrm{b}}$ & $284,7^{\mathrm{a}}$ & 3,1 \\
\hline $\begin{array}{l}\text { Dias em confinamento } \\
\text { Days on feedlot }\end{array}$ & $45,8^{\mathrm{C}}$ & $65,3^{\mathrm{b}}$ & $101,5^{\mathrm{a}}$ & 3,4 \\
\hline $\begin{array}{l}\text { Percentagem de traseiro especial } \\
\text { Yield of hindquarter gun cut, } \%\end{array}$ & $49,1^{\mathrm{a}}$ & $48,4^{\mathrm{a}}$ & $46,8^{\mathrm{b}}$ & 0,23 \\
\hline $\begin{array}{l}\text { Percentagem de dianteiro } \\
\text { Yield of forequarter cut, } \%\end{array}$ & $37,9^{b}$ & $38,7^{a}$ & $39,1^{\mathrm{a}}$ & 0,29 \\
\hline $\begin{array}{l}\text { Percentagem de ponta de agulha } \\
\text { Yield of flank with ribs cut, \% }\end{array}$ & $13,0^{\mathrm{a}}$ & $12,8^{\mathrm{a}}$ & $13,3^{\mathrm{a}}$ & 0,15 \\
\hline $\begin{array}{l}\text { Percentagem de traseiro total } \\
\text { Yield of hindquarter cut, \% }\end{array}$ & $62,1^{\mathrm{a}}$ & $61,3^{\mathrm{a}}$ & $60,1^{\mathrm{b}}$ & 0,29 \\
\hline $\begin{array}{l}\text { Traseiro esquerdo especial, kg } \\
\text { Left hindquarter gunbit cut, } \mathrm{kg}\end{array}$ & $55,7^{\mathrm{C}}$ & $60,9^{b}$ & $64,8^{\mathrm{a}}$ & 0,69 \\
\hline $\begin{array}{l}\text { Área de olho de lombo, } \mathrm{cm}^{2} \\
\text { Rib eye area, } \mathrm{cm}^{2}\end{array}$ & $74,1^{\mathrm{C}}$ & $81,6^{\mathrm{b}}$ & $87,8^{\mathrm{a}}$ & 1,78 \\
\hline
\end{tabular}

com os incrementos nos pesos de abate, de 400 para 440 e $480 \mathrm{~kg}$ nos animais Canchim e cruzados e de 375 para 385 e $400 \mathrm{~kg}$ nos animais Nelore, respectivamente. De maneira geral, era esperado que os aumentos nas porcentagem de gordura interna acarretassem redução nos rendimento de carcaça quente, já que no Brasil, diferentemente dos Estados Unidos (Sainz \& Araujo, 2001), esta fração do peso corporal não participa do peso de carcaça. Isto realmente ocorreu apenas para alguns animais que apresentaram valores elevados de gordura interna.

A AOL, uma medida da musculosidade da carcaça, quando expressa em $\mathrm{cm}^{2}$, sofreu influência $(\mathrm{P}<0,05)$ dos pesos de abate, apresentando também interação $(\mathrm{P}<0,05)$ entre GG e TRAT. Em todos GG cruzados (BN, CN, LN e PN), ocorreram aumentos $(\mathrm{P}<0,05)$ dos valores da AOL, com o aumento do peso de abate. Porém, quando os dados foram expressos em $\mathrm{cm}^{2} / 100 \mathrm{~kg}$ de carcaça, continuaram os efeitos de TRAT, mas a interação GG x TRAT deixou de ser significativa $(\mathrm{P}>0,05)$. As médias estimadas de 31,4; 30,9 e $30,2 \pm 0,31 \mathrm{~cm}^{2} / 100 \mathrm{~kg}$ de carcaça para os
TRAT I, II e III, respectivamente, demonstraram que os animais possuíam adequada conformação para produção de carne (acima de $30 \mathrm{~cm}^{2} / 100 \mathrm{~kg}$ de carcaça) e também se encontravam em fase de acabamento, isto é, aumento da musculatura menor $(\mathrm{P}<0,05)$ que aumento do peso de carcaça. Valores de AOL, expressos em $\mathrm{cm}^{2} / 100 \mathrm{~kg}$ de carcaça, entre 28,6 e 33,6, foram obtidos por Euclides Filho et al. (1997c), com animais NE entre 21,6 e 33,1 meses de idade. Resultados de outros autores concordam com os do presente trabalho, demonstrando redução linear da AOL de 27 para 24 e de 26,2 para 23,2 cm²/100 kg de carcaça, com o aumento do peso de abate de 550 para $700 \mathrm{~kg}$, em animais cruzados Blonde d'Aquitaine x Charolês (Patterson et al., 1994) e de 440 para 507 kg em animais cruzados Angus x Brahman (Huffman et al., 1990). Contudo, Galvão et al. (1991) e Jorge et al. (1997a) não encontraram redução na AOL, expressa em $\mathrm{cm}^{2} / 100 \mathrm{~kg}$ de peso corporal vazio, trabalhando com animais da raça Nelore, cruzados Limousin $x$ Nelore, Marchigiana x Nelore, Holandês x Nelore e bimestiço Fleckvieh x Angus x Nelore.

\section{R. Bras. Zootec., v.33, n.3, p.646-657, 2004}




\begin{tabular}{|c|c|c|c|c|}
\hline & & $\begin{array}{l}\text { de abat } \\
\text { hter weig }\end{array}$ & & $\begin{array}{r}\text { Erro-padrão } \\
\text { Standard error }\end{array}$ \\
\hline & 380 & 410 & 440 & \\
\hline $\begin{array}{l}\text { Peso de abate, } \mathrm{kg} \\
\text { Slaughter weight, } \mathrm{kg}\end{array}$ & $400,0^{\mathrm{C}}$ & $435,0^{\mathrm{b}}$ & $464,7^{\mathrm{a}}$ & 3,8 \\
\hline $\begin{array}{l}\text { Peso de carcaça quente, kg } \\
\text { Hot carcass weight, } \mathrm{kg}\end{array}$ & $235,9^{\mathrm{c}}$ & $253,8^{\mathrm{b}}$ & $276,7^{\mathrm{a}}$ & 2,0 \\
\hline $\begin{array}{l}\text { Dias em confinamento } \\
\text { Days on feedlot }\end{array}$ & $93,4^{\mathrm{b}}$ & $117,3^{\mathrm{a}}$ & $123,1^{\mathrm{a}}$ & 5,9 \\
\hline $\begin{array}{l}\text { Percentagem de traseiro especial } \\
\text { Yield of hindquarter gun cut, \% }\end{array}$ & $46,1^{\mathrm{a}}$ & $46,1^{\mathrm{a}}$ & $46,5^{\mathrm{a}}$ & 0,32 \\
\hline $\begin{array}{l}\text { Percentagem de dianteiro } \\
\text { Yield of forequarter cut, } \%\end{array}$ & $40,7^{\mathrm{a}}$ & $40,9^{\mathrm{a}}$ & $40,4^{\mathrm{a}}$ & 0,30 \\
\hline $\begin{array}{l}\text { Percentagem de ponta de agulha } \\
\text { Yield of flank with ribs cut, } \%\end{array}$ & $13,2^{\mathrm{a}}$ & $13,0^{\mathrm{a}}$ & $13,1^{\mathrm{a}}$ & 0,19 \\
\hline $\begin{array}{l}\text { Percentagem de traseiro total } \\
\text { Yield of hindquarter cut, } \%\end{array}$ & $59,3^{\mathrm{a}}$ & $59,1^{\mathrm{a}}$ & $59,6^{\mathrm{a}}$ & 0,30 \\
\hline Traseiro esquerdo especial, kg & $53,6^{\mathrm{C}}$ & $57,5^{b}$ & $63,1^{\mathrm{a}}$ & 0,57 \\
\hline $\begin{array}{l}\text { Área de olho de lombo, } \mathrm{cm}^{2} \\
\text { Rib eye area, } \mathrm{cm}^{2}\end{array}$ & $76,0^{\mathrm{C}}$ & $82,8^{\mathrm{b}}$ & $93,4^{\mathrm{a}}$ & 1,34 \\
\hline
\end{tabular}

1 Média estimada de 36 observações (Estimated means of 36 observations).

abc Médias seguidas de letras iguais, na mesma linha, não diferem $(P>0,05)$ pelo teste SNK.

abc Means in the same row, followed by the same letter, do not differ $(P>.05)$ by SNK test.

As médias estimadas da EGAOL, um indicador do ponto de terminação (acabamento) das carcaças, foram de 2,5; 3,1 e 3,6 $\pm 0,16 \mathrm{~mm}$ para os TRAT I, II e III, respectivamente. O aumento da EGAOL foi significativo $(\mathrm{P}<0,05)$ para os três pesos de abate, não ocorrendo interação significativa $(\mathrm{P}>0,05)$ entre TRAT e GG. Patterson et al. (1994) e Costa et al. (2002) observaram efeito linear para a espessura de gordura, com o aumento de peso de abate de 550 para $700 \mathrm{~kg}$, em animais cruzados Blonde d'Aquitaine $\mathrm{x}$ Charolês, e de 340 para 430 kg em novilhos Angus, respectivamente. Bailey et al. (1985) também observaram aumentos significativos da espessura de gordura em bovinos não-castrados da raça Holandesa, abatidos aos 340, 470 e 600 kg. Galvão et al. (1991) observaram aumento da EGAOL, expressa por $100 \mathrm{~kg}$ de peso corporal vazio, quando o peso de abate sofreu incrementos de 90 para 100 ou $110 \%$ do peso adulto do respectivo grupo genético. Porém, Jorge et al. (1997a) não encontraram diferença entre bovinos cruzados abatidos aos 500 e $550 \mathrm{~kg}$ e Nelore abatidos aos 450 e $500 \mathrm{~kg}$. Os frigoríficos que exportam carne resfriada adotam o sistema brasileiro de classificação e tipificação de carcaça (Sainz \& Araujo, 2001), avaliando subjetivamente, entre outros aspectos, o acabamento das carcaças, que são distribuídas em cinco categorias distintas: 1 - gordura ausente $(<1 \mathrm{~mm})$; 2 - gordura escassa (1 a $3 \mathrm{~mm}$ ); 3 - gordura mediana (3,1 a $6 \mathrm{~mm})$; 4 - gordura moderada (6,1 a $10 \mathrm{~mm})$; e 5 - gordura excessiva (>10 mm). Os valores entre parêntesis são apenas referências aproximadas para o avaliador e foram utilizados, neste trabalho, para calcular as porcentagens de animais tipificados como “B”, segundo o sistema brasileiro de classificação e tipificação de carcaça, discutido por Sainz \& Araujo (2001), baseando-se apenas no acabamento como parâmetro de classificação. As porcentagens de carcaças classificadas dentro das categorias de gordura 1, 2, 3 e 4 foram de 4,2; 77,5; 18,3 e 0,0 para o TRAT I; 7,0; 58,3; 33,3 e 1,4 para o TRAT II e 1,4; 52,8; 41,7 e 4,1 para o TRAT III, respectivamente, sendo que nenhum animal foi classificado na categoria de gordura 5 (excessiva). Os animais das categorias de gordura 1 e 5 são rebaixados da classificação "B" para "A” (dentro do padrão "B", "R", “A”, "S", "I” e "L”), ou seja, 4,2; 7,0 e 1,4\% dos animais dos TRAT 
Tabela 6 - Médias estimadas do peso de abate e características de carcaça de animais nãocastrados Nelore, abatidos aos 380 (I), 410 (II) e 440 (III) $\mathrm{kg}^{1}$

Table 6 - Estimated means of slaughter weight and carcass characteristics of Nellore bulls slaughtered at 380 (I), 410 (II) and 440 (III) $\mathrm{kg}^{1}$

\begin{tabular}{|c|c|c|c|c|}
\hline & \multicolumn{3}{|c|}{$\begin{array}{l}\text { Peso de abate, } \mathrm{kg} \\
\text { Slaughter weight, } \mathrm{kg}\end{array}$} & \multirow[t]{2}{*}{$\begin{array}{l}\text { Erro-padrão } \\
\text { Standard error }\end{array}$} \\
\hline & 380 & 410 & 440 & \\
\hline $\begin{array}{l}\text { Peso de abate, } \mathrm{kg} \\
\text { Slaughter weight, } \mathrm{kg}\end{array}$ & $376,9^{a}$ & $387,1^{\mathrm{a}}$ & $400,3^{a}$ & 7,5 \\
\hline $\begin{array}{l}\text { Peso de carcaça quente, } \mathrm{kg} \\
\text { Hot carcass weight, } \mathrm{kg}\end{array}$ & $211,8^{b}$ & $218,3^{\mathrm{ab}}$ & $228,2^{\mathrm{a}}$ & 4,1 \\
\hline $\begin{array}{l}\text { Dias em confinamento } \\
\text { Days on feedlot }\end{array}$ & $122,0^{C}$ & $138,3^{b}$ & $150,0^{\mathrm{a}}$ & 3,9 \\
\hline $\begin{array}{l}\text { Percentagem de traseiro especial } \\
\text { Yield of hindquarter gun cut, \% }\end{array}$ & $47,1^{\mathrm{a}}$ & $47,3^{\mathrm{a}}$ & $47,0^{\mathrm{a}}$ & 0,24 \\
\hline $\begin{array}{l}\text { Percentagem de dianteiro } \\
\text { Yield of forequarter cut, } \%\end{array}$ & $39,4^{\mathrm{a}}$ & $39,2^{\mathrm{a}}$ & $39,1^{\mathrm{a}}$ & 0,27 \\
\hline $\begin{array}{l}\text { Percentagem de ponta de agulha } \\
\text { Yield of flank with ribs cut, \% }\end{array}$ & $13,4^{\mathrm{a}}$ & $13,5^{\mathrm{a}}$ & $13,8^{\mathrm{a}}$ & 0,16 \\
\hline $\begin{array}{l}\text { Percentagem de traseiro total } \\
\text { Yield of hindquarter cut, \% }\end{array}$ & $60,6^{\mathrm{a}}$ & $60,8^{\mathrm{a}}$ & $60,8^{\mathrm{a}}$ & 0,26 \\
\hline $\begin{array}{l}\text { Traseiro esquerdo especial, kg } \\
\text { Left hindquarter gunbit cut, } \mathrm{kg}\end{array}$ & $49,4^{\mathrm{a}}$ & $50,7^{\mathrm{a}}$ & $52,4^{a}$ & 0,93 \\
\hline $\begin{array}{l}\text { Área de olho de lombo, } \mathrm{cm}^{2} \\
\text { Rib eye area, } \mathrm{cm}^{2}\end{array}$ & $60,8^{a}$ & $62,0^{\mathrm{a}}$ & $63,7^{\mathrm{a}}$ & 1,48 \\
\hline
\end{tabular}

1 Média estimada de 36 observações (Estimated means of 36 observations).

abc Médias seguidas de letras iguais, na mesma linha, não diferem $(P>0,05)$ pelo teste SNK.

abc Means in the same row, followed by the same letter, do not differ $(P>.05)$ by SNK test.

I, II e III foram desclassificados por apresentarem gordura ausente. Nenhum animal NE foi desclassificado por apresentar padrão de gordura ausente, e sim por apresentar baixo peso de carcaça, como já foi discutido anteriormente. As portarias das Secretarias de Agricultura dos diversos Estados que instituíram incentivos à produção do bovino jovem (Mattos, 1995; Pires, 1995) também adotaram este critério de acabamento das carcaças. Contudo, em algumas alianças mercadológicas no Estado de São Paulo, alguns frigoríficos adotaram os limites de 3 a $10 \mathrm{~mm}$ em um padrão próprio para classificar as carcaças, desclassificando então as carcaças com gordura escassa. Nesta norma mais rígida de classificação das carcaças, pode-se observar que a maioria dos animais NE (83,3\%) de qualquer peso de abate e CN dos TRAT II e III e CA do TRAT III possuía pelo menos $3 \mathrm{~mm}$ de espessura de gordura externa. Para os grupos genéticos LN e PN, 58,3\% dos animais com $480 \mathrm{~kg}$ de peso vivo (TRAT III) possuíam pelo menos $3 \mathrm{~mm}$ de gordura externa, enquanto, no grupo genético $\mathrm{BN}$, apenas $38,9 \%$, indicando que, nos cruzamentos com raças européias continentais de musculatura bastante desenvolvida, existe necessidade de testar pesos de abate dos machos não-castrados acima de $480 \mathrm{~kg}$, visando o abate de animais com $5 \mathrm{~mm}$ de cobertura de gordura, conforme sugerido por Euclides Filho et al. (2001).

O rendimento da porção comestível do TEE, obtida pela desossa tradicional, expresso em porcentagem do peso do traseiro, não sofreu influencia $(\mathrm{P}>0,05)$ dos pesos de abate e da interação GG x TRAT. Os resultados estão de acordo com aqueles obtidos por Patterson et al. (1994), trabalhando com animais cruzados Blonde d'Aquitaine x Charolês e peso de abate de 550 a $700 \mathrm{~kg}$; os quais obtiveram rendimento de desossa de $70,7 \%$, para a carcaça inteira. Por outro lado, Levy et al. (1975) encontraram aumento no rendimento de carne comercializável de 74,8 para 75,9 ou 76,1\% com o aumento de peso de abate de animais da raça Holandesa de 400 para 450 ou $500 \mathrm{~kg}$. Urick et al. (1991) encontraram 68,8 e $66,5 \%$ de carne comercializável na carcaça de bovinos castrados cruzados de diferentes raças com Hereford, quando os pesos de abate foram definidos como sendo peso fixo de $400 \mathrm{~kg}$ sem jejum ou composição corporal fixa de $12,7 \mathrm{~mm}$ de gordura externa nas carcaças. Huffman et al. (1990) encon- 
Tabela 7 - Médias estimadas de idade de abate, dias em confinamento e características de carcaça de animais não-castrados abatidos aos 400 (I), 440 (II) e 480 (III) kg ${ }^{1}$

Table 7 - Estimated means of age of slaughter, days on feedlot and carcass characteristics of bulls slaughtered at 400 (I), 440 (II) and 480 (III) $\mathrm{kg}^{1}$

\begin{tabular}{|c|c|c|c|c|}
\hline & \multicolumn{3}{|c|}{$\begin{array}{l}\text { Peso de abate, kg } \\
\text { Slaughter weight, kg }\end{array}$} & \multirow[t]{2}{*}{$\begin{array}{l}\text { Erro-padrão } \\
\text { Standard error }\end{array}$} \\
\hline & 380 & 410 & 440 & \\
\hline Idade de abate, meses & $15,5^{\mathrm{c}}$ & $16,3^{\mathrm{b}}$ & $16,9^{\mathrm{a}}$ & 0,15 \\
\hline Dias em confinamento & $70,9^{\mathrm{c}}$ & $95,1^{b}$ & $114,7^{\mathrm{a}}$ & 2,1 \\
\hline $\begin{array}{l}\text { Rendimento de carcaça quente, \% } \\
\text { Yield of hot carcass, \% }\end{array}$ & $57,2^{\mathrm{b}}$ & $57,7^{\mathrm{b}}$ & $58,3^{\mathrm{a}}$ & 0,19 \\
\hline Rendimento de carcaça resfriada, \% & $56,8^{b}$ & $57,1^{\mathrm{ab}}$ & $57,5^{\mathrm{a}}$ & 0,19 \\
\hline $\begin{array}{l}\text { Gordura peri-renal, pélvica, inguinal, \% } \\
\text { Perinephric and retroperitoneal fat, \% }\end{array}$ & $3,2^{b}$ & $3,6^{\mathrm{a}}$ & $3,7^{\mathrm{a}}$ & 0,09 \\
\hline $\begin{array}{l}\text { Área de olho de lombo, } \mathrm{cm}^{2} / 100 \mathrm{~kg} \text { de carcaça } \\
\text { Rib eye area, } \mathrm{cm}^{2} / 100 \mathrm{~kg} \text { carcass weight }\end{array}$ & $31,4^{\mathrm{a}}$ & $30,9^{\mathrm{ab}}$ & $30,2^{\mathrm{b}}$ & 0,31 \\
\hline Espessura de gordura externa, mm & \multicolumn{4}{|c|}{ Back fat thickness, $\mathrm{mm}$} \\
\hline $\begin{array}{l}\text { Carne comestível do } \mathrm{TEE}^{2}, \% \\
\text { Hindquarter gunbit saleable meat, \% }\end{array}$ & $73,1^{\mathrm{a}}$ & $73,0^{\mathrm{a}}$ & $73,2^{\mathrm{a}}$ & 0,20 \\
\hline $\begin{array}{l}\text { Ossos do TEE }{ }^{2}, \% \\
\text { Bones in hindquarter gunbit cut, \% }\end{array}$ & $18,4^{\mathrm{a}}$ & $18,2^{\mathrm{ab}}$ & $17,9^{\mathrm{b}}$ & 0,14 \\
\hline $\begin{array}{l}\text { Bones in hindquarter gunbit cut, } \% \\
\text { Retalho gordo (aparas) do TEE }{ }^{2}, \% \\
\text { Fat trim in hindquarter gunbit cut, } \%\end{array}$ & $8,0^{\mathrm{b}}$ & $8,6^{\mathrm{a}}$ & $8,7^{\mathrm{a}}$ & 0,15 \\
\hline \multicolumn{5}{|c|}{$\begin{array}{l}\text { Média estimada de } 215 \text { observações de seis grupos genéticos (BN; CN; CA; LN, NE, PN), } \\
\text { animais NE foram abatidos com } 380,410 \text { e } 440 \mathrm{~kg} \text { (Estimated means of } 215 \text { observations of six ger } \\
\text { (BN; CN; CA; LN, NE, PN), except that NE cattle were slaughtered at } 380,410 \text { and } 440 \mathrm{~kg}) \text {. } \\
\text { Traseiro esquerdo especial (Left hindquarter gunbit cut). } \\
\text { abc Médias seguidas de letras iguais, na mesma linha, não diferem }(P>0,05) \text { pelo teste SNK. } \\
\text { abc Means in the same row, followed by the same letter, do not differ }(P>05) \text { by SNK test. }\end{array}$} \\
\hline
\end{tabular}

traram redução do rendimento de desossa de 2,5 para 3,5 numa escala de 1 (maior) a 5 (menor), quando o peso de abate de animais cruzados Angus x Brahman aumentaram de 440 para $507 \mathrm{~kg}$. Junqueira et al. (1998) obtiveram 75,0\% de carne aproveitável no traseiro especial de bovinos não-castrados cruzados Marchigiana x Nelore.

As médias estimadas das percentagens de ossos do TEE foram 18,4; 18,2 e 17,9 $\pm 0,14$ para os tratamentos I, II e III, respectivamente, apresentando diferenças $(P<0,05)$ entre TRAT, ocorrendo redução significativa da porcentagem de ossos, à medida que aumentou o peso de abate. Estes resultados estão de acordo com aqueles obtidos por Patterson et al. (1994), Levy et al. (1975) e Breidenstein et al. (1965), citados por Preston \& Willis (1974), que encontraram redução na porcentagem de ossos na carcaça inteira, com incrementos no peso de abate. No trabalho citado por Preston \& Willis (1974), ocorreram reduções nas percentagens de ossos na carcaça inteira de
17,6 para 16,$4 ; 14,9$ ou $13,5 \%$, quando os pesos de abate aumentaram de 307 para 386, 466 ou 545 kg, respectivamente.

As médias estimadas de aparas de gordura (retalho gordo) do TEE foram 8,0; 8,6 e 8,7\% para os TRAT I, II e III, respectivamente, apresentando diferenças $(\mathrm{P}<0,05)$ entre TRAT. Ocorreram aumentos das percentagens de aparas de gordura, à medida que aumentou o peso de abate, mostrando efeitos significativos $(\mathrm{P}<0,05)$ entre os TRAT I e II. $\mathrm{O}$ aumento das aparas de gordura entre tratamentos foi pequeno, se considerar o fato de que a maioria das carcaças foi classificada como magra (baixa cobertura de gordura externa). Estes resultados estão de acordo com aqueles obtidos por Patterson et al. (1994) e Levy et al. (1975), que encontraram aumento na percentagem de aparas de gordura na carcaça inteira com incrementos no peso de abate, enquanto Kropf \& Graf (1959), citados por Preston \& Willis (1974), encontraram aumentos na porcentagem de

R. Bras. Zootec., v.33, n.3, p.646-657, 2004 
aparas de gordura de 14,9 para 17,8 ou $20,7 \%$ nas carcaças classificadas como comercial, boa ou escolha, no sistema norte-americano de tipificação de carcaças, descrito por (Sainz \& Araujo, 2001). Este sistema se baseia, entre outros fatores, na quantidade de gordura intramuscular (marmorização) avaliada no corte transversal do músculo Longissimus. A marmorização dos tecidos ocorre após deposição de elevada quantidade de gordura, tanto interna (perirenal, inguinal) quanto subcutânea, o que explica os valores elevados de porcentagem de aparas de gordura nas carcaças. Por outro lado, em trabalhos realizados dentro do sistema tradicional de desossa no Brasil, Junqueira et al. (1998) obtiveram 8,54\% de aparas de gordura no traseiro especial de bovinos não-castrados cruzados Marchigiana x Nelore.

\section{Conclusões}

Nas condições do presente estudo, o peso de abate de $440 \mathrm{~kg}$ é adequado para animais $1 / 2$ Canchim x $1 / 2$ Nelore não-castrados, enquanto o peso de 480 kg deve ser utilizado para animais Canchim, para obtenção de carcaças com pelo menos $3 \mathrm{~mm}$ de cobertura de gordura externa.

O abate de animais $1 / 2\left(3 /{ }_{8}\right)$ Blonde d'Aquitaine X $1 / 2(5 / 8)$ Nelore, $1 / 2$ Limousin $x$ 1/2 Nelore e $1 / 2$ Piemontês $x$ 1/2 Nelore com pesos acima de $480 \mathrm{~kg}$, para obtenção de carcaças com boa cobertura de gordura externa, é adequado.

Outras alternativas de alimentação e manejo necessitam ser estudadas para os animais da raça Nelore comercial, tendo em vista que o confinamento de animais com 12 meses de idade e $213 \mathrm{~kg}$ de peso vivo inicial mostra-se inviável, uma vez que produz animais acabados com peso abaixo do desejado pelo mercado.

Os pesos de abate mais elevados produzem carcaças com menor proporção de traseiro especial, desvalorizando a carcaça como um todo no mercado.

\section{Agradecimento}

Aos produtores rurais Amilcar Farid Yamim, Antônio Villares da Silva Novaes, José Ferreira Fernandes e Rubens de Assumpção, pela adesão ao contrato de parceria para realização do confinamento, e ao Frigorífico Angelelli Ltda., pela permissão para coleta de dados de abate e qualidade de carcaça.

\section{Literatura Citada}

ABULARACH, M.L.S.; ROCHA, C.E.; FELICIO, P.E. Características da qualidade do contrafilé (m. L. dorsi) de touros jovens da raça Nelore. Ciência Tecnologia Alimentos, v.18, n.2, p.205-210, 1998.

BAILEY, C.M.; LIBORIUSSEN, T.; ANDERSEN, H.R. et al. Producing beef from intact male progeny of Holstein sires: feed efficiency and compositional characters. Journal of Animal Science, v.61, n.1, p.27-35, 1985.

BARBER, K.A.; WILSON, L.L.; ZIEGRER, J.H. et al. Charolais and angus steers slaughtered at equal percentages of mature cow weight. I. Effects of slaughter weight and diet energy density on carcass traits. Journal of Animal Science, v.52, n.2, p.218-231, 1981.

BROADBENT, P.J. Growth and carcass characteristics of weaned single-suckled calves slaughtered on achieving slaughter condition or 8 or 16 weeks later. Animal Production, v.23, n.2, p.155-163, 1976.

COSTA, E.C.; RESTLE, J.; VAZ, F.N. et al. Características da carcaça de novilhos Red Angus superprecoces abatidos com diferentes pesos. Revista Brasileira de Zootecnia, v.31, n.1, p.119-128, 2002.

CROUSE, J.D.; CUNDIFF, L.V.; KOCH, R.M. et al. Comparison of Bos Indicus and Bos Taurus inheritance for carcass beef characteristics and meat palatability. Journal of Animal Science, v.67, n.10, p.2661-2668, 1989.

EUCLIDES FILHO, K.; EUCLIDES, V.P.B.; FIGUEIREDO, G.R. et al. Avaliação de animais Nelore e de seus mestiços com Charolês, Fleckvieh e Chianina, em três dietas.1. Ganho de peso e conversão alimentar. Revista Brasileira de Zootecnia, v.26, n.1, p.66-72, 1997a.

EUCLIDES FILHO, K.; EUCLIDES, V.P.B.; FIGUEIREDO, G.R. et al. Avaliação de animais Nelore e de seus mestiços com Charolês, Fleckvieh e Chianina, em três dietas. 2. Caracteristicas de carcaça. Revista Brasileira de Zootecnia, v.26, n.1, p.73-79, 1997b.

EUCLIDES FILHO, K.; EUCLIDES, V.P.B.; FIGUEIREDO, G.R. et al. Efeito da suplementação com concentrado sobre idade de abate e características de carcaça de bovinos Nelore. Revista Brasileira de Zootecnia, v.26, n.6, p.1096-1102, 1997c.

EUCLIDES FILHO, K.; FEIJÓ, G.L.D.; FIGUEIREDO, G.R. et al. Efeito da idade à castração e de grupos genéticos sobre o desempenho em confinamento e características de carcaça. Revista Brasileira de Zootecnia, v.30, n.1, p.71-76, 2001.

FELÍCIO, P.E. Fatores ante e post mortem que influenciam na qualidade da carne bovina. In: SIMPÓSIO SOBRE PECUÁRIA DE CORTE (Produção do novilho de corte), 4., 1996, Piracicaba. Anais... Piracicaba: Fundação de Estudos Agrários Luiz de Queiroz, 1997. p. 79-97.

FRANCIS, S.J.; ALLEN, D.M.; KASTNER, C.L. et al. The effect of coring method of beef Longissimus muscle shear force values. Journal of Animal Science, v.52, n.6, p.1294-1297, 1981.

GALVÃO, J.G.; FONTES, C.A.A.; PIRES, C.C. et al. Características e composição física da carcaça de bovinos nãocastrados, abatidos em três estágios de maturidade (Estudo II) de três grupos raciais. Revista da Sociedade Brasileira de Zootecnia, v.20, n.5, p.502-512, 1991.

HUFFMAN, R.D.; WILLIAMS, S.E.; HARGROVE, D.D. et al. Effects of percentage Brahman and Angus breeding, age- 
season of feeding and slaughter end point on feedlot performance and carcass characteristics. Journal of Animal Science, v.68, n.8, p.2243-2252, 1990.

JORGE, A.M.; FONTES, C.A.A.; SOARES, J.E. et al. Características quantitativas da carcaça de bovinos e bubalinos, abatidos em diferentes estágios de maturidade. Revista Brasileira de Zootecnia, v.26, n.5, p.1039-1047, 1997a.

JORGE, A.M.; FONTES, C.A.A.; FREITAS, J.A. et al. Rendimento da carcaça e de cortes básicos de bovinos e bubalinos, abatidos em diferentes estágios de maturidade. Revista Brasileira de Zootecnia, v.26, n.5, p.1048-1054, 1997 b.

JUNQUEIRA, J.O.B.; VELLOSO, L.; FELÍCIO, P.E. Desempenho, Rendimento de carcaça e cortes de animais, machos e fêmeas, mestiços Marchigiana x Nelore, terminados em confinamento. Revista Brasileira de Zootecnia, v.27, n.6, p.1199-1205, 1998.

LEVY, D.; HOLZER, Z.; FOLMAN, Y. Effect of concentrate: roughage ratio on the production of beef from israeli-friesian bulls slaughtered at different live weights. Animal Production, v.20, n.3, p.199-205, 1975.

MATTOS, J.C.A. Programa de produção de carne qualificada de bovídeos do Estado de São Paulo (Novilho Precoce). In: ENCONTRO NACIONAL SOBRE NOVILHO PRECOCE, 1., 1995, Campinas. Anais... Campinas: Coordenadoria de Assistência Técnica Integral, 1995. p.13-21.

PATTERSON, D.C.; MOORE, C.A.; STEEN, R.W.J. The effects of plane of nutrition and slaughter weight on the performance and carcass composition of continental beef bulls given high forage diets. Animal Production, v.58, n.1, p.41-47, 1994.
PIRES, J.A.A. Programa estadual de apoio à produção de novilho precoce de Minas Gerais. In: ENCONTRO NACIONAL SOBRE NOVILHO PRECOCE, 1., 1995, Campinas. Anais... Campinas: Coordenadoria de Assistência Técnica Integral, 1995. p.25-38.

PEROTTO, D.; ABRAHÃO, J.J.S.; MOLETTA, J.L. Características quantitativas de carcaça de bovinos zebu e de cruzamentos Bos Taurus x zebu. Revista Brasileira de Zootecnia, v.29, n.6, p.2019-2029, 2000 (suplemento 1).

PRESTON, T.R.; WILLIS, M.B. Intensive beef production. 2.ed. Oxford: Pergamon Press, 1974. 567p.

SAINZ, R.D.; ARAUJO, F.R.C. Tipificação de carcaças de bovinos e suínos. In: CONGRESSO BRASILEIRO DE CIÊNCIA E TECNOLOGIA DE CARNES, 1., 2001, São Pedro. Anais... Campinas: Centro de Tecnologia de Carnes do Instituto de Tecnologia de Alimentos, 2001. p.26-55.

STATISTICAL ANALYSES SYSTEM - SAS. User's guide: statistics. Cary: 1999. Site 0030966035.

URICK, J.J.; MacNEIL, M.D.; REYNOLDS, W.L. Biological type on postweaning growth, feed efficiency and carcass characteristics of steers. Journal of Animal Science, v.69, n.2, p.490-497, 1991.

Recebido em: 05/09/02

Aceito em: 10/09/03 